\title{
Texture Based Automated Segmentation of Skin Lesions using Echo State Neural Networks
}

\section{Z. Faizal Khan ${ }^{\dagger}$ and Nalinipriya Ganapathi*}

\begin{abstract}
A novel method of Skin lesion segmentation based on the combination of Texture and Neural Network is proposed in this paper. This paper combines the textures of different pixels in the skin images in order to increase the performance of lesion segmentation. For segmenting skin lesions, a two-step process is done. First, automatic border detection is performed to separate the lesion from the background skin. This begins by identifying the features that represent the lesion border clearly by the process of Texture analysis. In the second step, the obtained features are given as input towards the Recurrent Echo state neural networks in order to obtain the segmented skin lesion region. The proposed algorithm is trained and tested for 862 skin lesion images in order to evaluate the accuracy of segmentation. Overall accuracy of the proposed method is compared with existing algorithms. An average accuracy of $98.8 \%$ for segmenting skin lesion images has been obtained.
\end{abstract}

Keywords: Image segmentation, Texture analysis, Skin lesion, Echo State Neural network (ESNN)

\section{Introduction}

Skin Cancer is the deadliest form of cancer, with an estimated 76690 people being diagnosed with melanoma and 9480 people dying of melanoma in the United States in 2013. In the United States, the lifetime risk of getting melanoma is 1 in 49 [1]. Melanoma accounts for approximately $75 \%$ of deaths associated with melanoma. It is a malignant lesion of the melanocytes and usually occurs on the trunk or lower extremities [2]. Dermoscopy is a noninvasive skin imaging technique that uses optical magnification to make the contact area translucent, making subsurface structures more easily visible when compared to conventional clinical images. With the rising occurrence rates in certain subsets of the general population, it is useful to screen for melanoma in order to detect it in earlier stage. Hence an automated skin lesion segmentation algorithm has been proposed in order to increase the survival rate. Recent literatures with automated skin cancer segmenting methods tries to adapt the algorithms for analysing images taken by a standard digital camera [7]. A new method for segmenting skin images using the texture descriptiveness (TD) and Neural Networks is proposed by jisha et al in [15]. In their methodology, the TD captures the dissimilarity between different texture distributions present in the images.

Regions in the image are classified as based on TD metric. In this TD algorithm, the image is converted into $\mathrm{XYZ}$ color space. A TD metrics is calculated for each

$\dagger \quad$ Corresponding Author: Dept. of Computer and Network Engineering, College of Engineering, Shaqra University, Kingdom of Saudi Arabia. (faizalkhan111@yahoo.co.in)

* Department of Information Technology, Saveetha Engineering College, Chennai, Tamilnadu., India.

Received: July 15, 2015; Accepted: October 7, 2016 texture distribution based on the probability of it being similar to other texture distributions [8]. This information is combined with the contents of each region to determine a regional TD metric. The main difference between the proposed method and this method is the usage of GLCM features instead of Texture descriptiveness. The majority of algorithms only use features derived from pixel color to drive the segmentation. Stoecker et al. [18] proposed a novel texture based algorithm in skin images using basic statistical approaches such as the gray-level co-occurrence matrix and region merging [8]. From their algorithm, they found that texture analysis could accurately find regions with a smooth texture and it can be applicable for segmentation and classification of skin lesion images.

A modified algorithm proposed by Xu et al. [19] trains a model of the normal skin texture using pixels in the four corners of the skin lesion image in order to find the lesion present in it.

Jeffrey et al [1] proposed a new methodology for segmenting and classifying the skin lesion images. They segmented the lesion present in the skin images Using Joint Statistical Texture Distinctiveness method which results in accuracy of $93.28 \%$ of segmentation. A Combination of several image processing techniques with Support Vector Machine was proposed in [20]. Initially the skin border was detected by JSEG algorithm. It included three main phases' namely preprocessing, segmentation, and post processing. The preprocessing phase included image smoothing using a color median filter, color reduction using the variance-based quantization method and approximate lesion localization based on the Otsu thresholding method. Then the features are given as input to the Support Vector Machine in order to segment the optimal skin lesion region. An average specificity of $92.34 \%$ and a sensitivity 


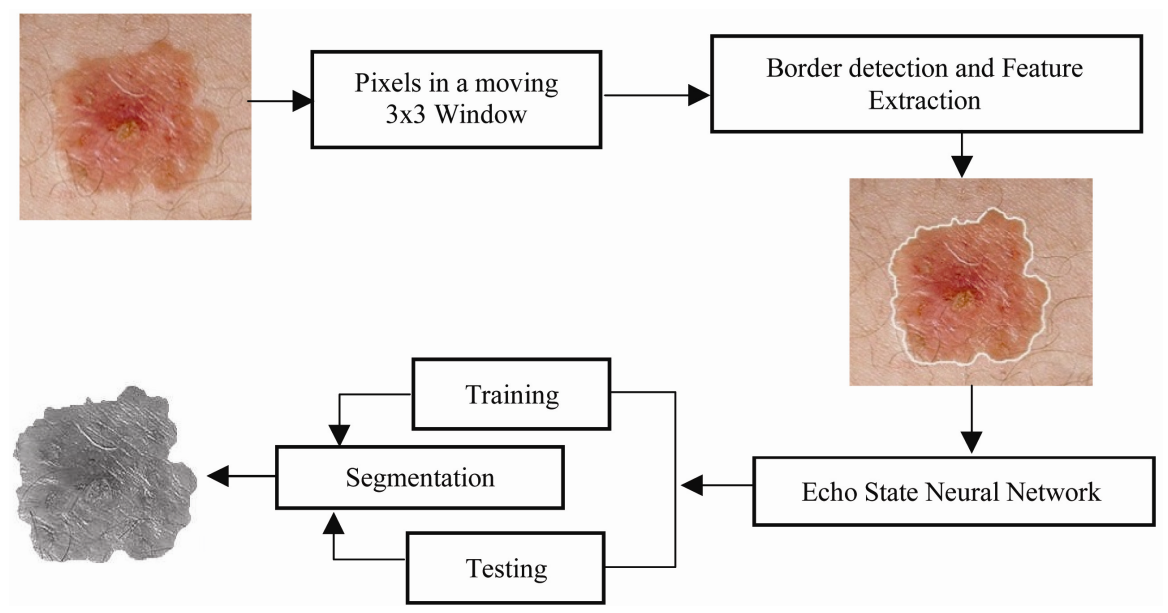

Fig. 1. Architecture of the proposed segmentation algorithm.

of $93.33 \%$ were obtained for 564 skin lesion images.

A combination of Semi-automatic and manual method was proposed by Menzies et al in [22]. They used $n$ Logistic regression based classifier for classifying the segmented lesions. They applied their algorithm for 2430 skin lesion images and obtained an average specificity of $65 \%$ and a sensitivity of $91 \%$. Stolz et al [23] introduced a simplified the dermoscopic diagnosis of melanocytic skin lesions by modifying the ABCD rule used for clinical diagnosis, known as the ABCD rule of dermoscopy (asymmetry, borders, colors and dermoscopic structures). This method adopts a scoring system that permits the diagnosis of melanoma. Nachbar et al. modified this method in a prospective study showing a specificity of $90 \%$ and a sensitivity of $93 \%$ in the diagnosis of melanoma [24]. From the works found in the above literature, it has been observed that most of the existing works shows less accuracy in segmenting skin lesion images. Therefore, it is necessary to propose a new and efficient technique to segment the skin lesions.

\section{Materials and Methods}

\subsection{Border detection}

The first step in the computerized segmentation of skin lesion images is the identification of the lesion borders. For border detection, an automated unsupervised border detection method $[3,16]$ was used. The overall architecture of the proposed methodology is illustrated in Fig. 1.

\subsection{Feature extraction}

In this section, the features that were used to characterize the skin lesion from the outer structure is described. Texture provides some important features about the structural arrangement of various surfaces. Texture features are used to classify the possible sites of nodules that have been identified with previous processing [5]. Gray Level CoOccurrence Matrix (GLCM) features are calculated for the regions present inside the border of the shin lesion image. The fourteen textural features proposed by Haralick et al [4] contains the information about image texture and its characteristics. In general, GLCM creates grey-co matrix by calculating the frequency with which a pixel with greylevel (greyscale intensity) value ' $i$ ' occurs horizontally adjacent to a pixel with the value ' $j$ '. Each element $(i, j)$ in GLCM specifies the number of times that the pixel with value ' $i$ ' occurs horizontally adjacent to a pixel with value ' $j$ '. Subsequently, the other features such as correlation, energy, contrast, homogeneity are obtained. The obtained features are given as input towards the Recurrent Echo state neural networks (ESNN) for training in order to obtain the segmented lesion region

\subsection{ESNN with texture topology}

Echo state network $[13,14]$ is a recurrent discrete-time neural network with $\mathrm{S}$ input units, $\mathrm{X}$ internal (reservoir) units, and Y output units. Connection weights and the input weights in the ESNN reservoir are randomly generated. A typical echo state neural network has three layers such as an input layer, middle layer and an output layer. The activation function $[25,21]$ of the input layer, middle layer and the output layers at time $\mathrm{t}$ can be calculated by the following equation.

$$
\begin{aligned}
\mathrm{W}_{\mathrm{S}(\mathrm{t})} & =\left(\mathrm{s}_{1}(\mathrm{t})+\mathrm{s}_{2}(\mathrm{t})+\ldots . . \mathrm{s}_{\mathrm{n}}(\mathrm{t})\right) \\
\mathrm{W}_{\mathrm{X}(\mathrm{t})} & =\left(\mathrm{x}_{1}(\mathrm{t})+\mathrm{x}_{2}(\mathrm{t})+\ldots . . \mathrm{x}_{\mathrm{n}}(\mathrm{t})\right) \\
\mathrm{W}_{\mathrm{Y}(\mathrm{t})} & =\left(\mathrm{y}_{1}(\mathrm{t})+\mathrm{y}_{2}(\mathrm{t})+\ldots . . \mathrm{y}_{\mathrm{n}}(\mathrm{t})\right)
\end{aligned}
$$

The connections between the input units and the internal units are given by an $\mathrm{S} \times \mathrm{X}$ weight matrix $\mathrm{V}$, connections between the internal units are collected in an $\mathrm{X} \times \mathrm{X}$ weight matrix $\mathrm{W}$, and connections from internal units to output units are given in $\mathrm{X} \times \mathrm{Y}$ weight matrix $\mathrm{U}$ [10]. The input units are updated based on the following equation 


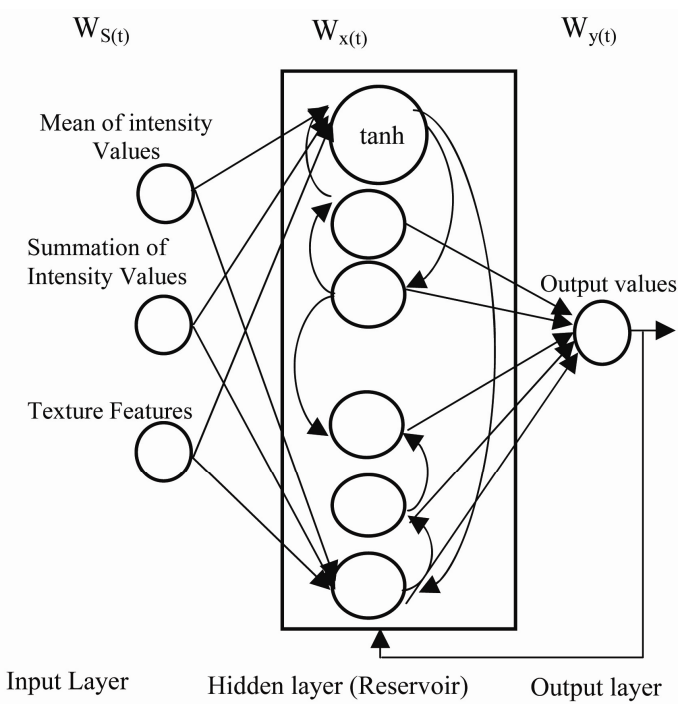

Fig. 2. Proposed ESNN with texture topology

$$
\mathrm{S}(\mathrm{t}+1)=f(\mathrm{Vs}(\mathrm{t}+1)+\mathrm{Wx}(\mathrm{t}))
$$

Where, $f$ is the reservoir activation function which is a "tanh" sigmoidal function. The output units are updated based on the following equation

$$
\mathrm{Y}(\mathrm{t}+1)=f(\mathrm{Uy}(\mathrm{t}+1)+\mathrm{Wx}(\mathrm{t}))
$$

The nodes between the input layer and the hidden layer are connected such that the output node from the output layer is fed back towards the hidden layer. The Proposed topology in Fig. 2 consists of three layers. The layer 1 is the input layer $\left(\mathrm{W}_{\mathrm{S}(\mathrm{t})}\right)$. Layer 2 is the hidden layer $\left(\mathrm{W}_{\mathrm{X}(\mathrm{t})}\right)$ and layer 3 is the output layer $\left(\mathrm{W}_{\mathrm{Y}(\mathrm{t})}\right)$ which contains reservoirs (nodes).

\subsubsection{Working principle of ESNN}

The ESNN is trained with training patterns obtained from texture analysis in order to obtain the final weights. During the testing of ESNN algorithm, a pattern from the moving window is processed with already trained weights to obtain an output in the output layer of ESNN. The training and testing procedures are given below.

\section{Training ESNN}

In order to segment the image, the three features of the windows are presented in the input layer and the corresponding target outputs $(0.1$ or 0.9$)$ are presented in the output layer of the ESNN. A state vector is initialized with zero. The length of the state vector is equivalent to the number of nodes or reservoirs in the hidden layer of the ESNN.

The number of reservoirs [26, 27] is decided based on the minimum segmentation error obtained in the segmented image. A new state vector is obtained by passing the summed value over an activation 'tanh'. Hence, 100 state
Step 1: Input the texture features obtained from 862 images.

Step 2: Decide the number of reservoirs.

Step 3: Decide the number of nodes in the input layer $=3$.

Step 4: Decide the number of nodes in the output layer $=$ number of target values.

Step 5: Initialize state vector (number of reservoirs) $=0$.

Step 6: Initialize random weights between input layer $\left(\mathrm{W}_{\mathrm{S}(\mathrm{t})}\right)$ and hidden layer $\left(\mathrm{W}_{\mathrm{X}(\mathrm{t})}\right)$. Initialize weights between output layer $\left(\mathrm{W}_{\mathrm{Y}(\mathrm{t})}\right)$ and hidden layer $\left(\mathrm{W}_{\mathrm{X}(\mathrm{t})}\right)$. Initialize weights in the reservoirs.

Step 7:Calculate state_vector ${ }_{\text {next }}=$ $\tanh \left(\left(\mathrm{W}_{\mathrm{S}(\mathrm{t})} \mathrm{W}_{\mathrm{X}(\mathrm{t})}\right)_{\text {weights }}{ }^{*}\right.$ Input $_{\text {pattern }}+\left(\mathrm{W}_{\mathrm{X}(\mathrm{t})}\right)_{\text {weights }} *$ state vector ${ }_{\text {present }}+\left(\mathrm{W}_{\mathrm{X}(\mathrm{t})} \mathrm{W}_{\mathrm{Y}(\mathrm{t})}\right)_{\text {weights }} *$ Target $\left._{\text {pattern }}\right)$.

Step 8: Calculate, $\mathrm{a}=$ Pseudo inverse (State vector ${ }_{\text {all patterns }}$ ).

Step 8: Calculate, $\mathrm{W}_{\text {out }}=\mathrm{a} * \mathrm{~T}$ and store $\mathrm{W}_{\text {out }}$ for segmentation.

vectors are obtained if there are 100 training patterns. An ESNN matrix is obtained whose size is number of training patterns (100) X number of reservoirs (22). This matrix is a rectangular matrix and hence a pseudo inverse of the ESNN matrix is found and multiplied with the target values to obtain final weights.

\section{Testing ESNN}

The Texture features obtained from the 862 images are given as input to the input layer of the ESNN. The algorithm for testing ESNN is proposed in algorithm is shown below. The output of ESNN is obtained from step 3 . The final value is compared with a threshold of 0.5 . If the total value is $<=0.5$, then 0 is assigned to the centre of window else 1 assigned.

Algorithm for Testing or segmenting the image

Step 1: Input the Texture features obtained from 862 images

Step 2: Decide the number of reservoirs

Step 3: Calculate state vector $=\tanh \left(\mathrm{W}_{\mathrm{S}(\mathrm{t})} \mathrm{W}_{\mathrm{X}(\mathrm{t})}\right)_{\text {weights }} * \operatorname{Input}_{\text {pattern }}+\left(\mathrm{W}_{\mathrm{X}(\mathrm{t})}\right)_{\text {weights }} *$ state vector present $+\left(\mathrm{W}_{\mathrm{X}(\mathrm{t})} \mathrm{W}_{\mathrm{Y}(\mathrm{t})}\right)_{\text {weights }} *$ Target $\left._{\text {pattern }}\right)$.

Step 4: Estimated output $=$ state vector $* \mathrm{~W}_{\text {out }}$.

Step 5: Assign '0' (black) or ' 255 ' (white) in the new matrix which will be the segmented image.

Step 6: The resulting image is the desired segmented output

\section{Results and Discussion}

Accuracy of segmentation has been evaluated in order to assess the performance of the proposed segmentation algorithm. In this section, we present the results of the proposed methodology in terms of qualitatively and 
quantitatively through image display and experimental analysis respectively. The proposed approach was applied to the initial set of 862 images. All the 862 images were used for the process of training as well as testing. Table 3 shows examples of different skin lesion detection results.

\subsection{Segmentation Performance in Terms of sensiti- vity, specificity, and accuracy}

The segmentation accuracy based on sensitivity, specificity, and accuracy [17]. The proposed algorithm is applied to various skin lesion images taken from the Dermquest database [9] and the segmentation results are

Table 1. Segmentation accuracy based on sensitivity, specificity and accuracy

\begin{tabular}{c|c|c|c}
\hline Image & Sensitivity & Specificity & Accuracy \\
\hline Image 1 & 91.4 & 99.5 & 98.7 \\
\hline Image 2 & 90.4 & 98.8 & 98.6 \\
\hline Image 3 & 91.3 & 97.8 & 99.6 \\
\hline Image 4 & 90.5 & 99.7 & 98.6 \\
\hline Image 5 & 90.2 & 99.8 & 98.5 \\
\hline Average & 90.6 & 99.2 & 98.8 \\
\hline
\end{tabular}

compared to the manually drawn images called ground truth. The metrics used to compare to the ground truth are sensitivity, specificity, and accuracy. Their formulas are as follows.

$$
\begin{gathered}
\text { Sensitivity }=\frac{T P}{T P+F N} \\
\text { Specificity }=\frac{T N}{T N+F P} \\
\text { Accuracy }=\frac{T P+T N}{T P+F N+T N+F P}
\end{gathered}
$$

\begin{tabular}{|c|c|c|c|c|c|c|}
\hline Image & Region & Solidity & Area & Perimeter & Total & Aprop \\
\hline \multirow{2}{*}{$\begin{array}{c}\text { Image } \\
1\end{array}$} & Segmented & \begin{tabular}{|l|}
154.1526 \\
\end{tabular} & 1648 & 726.3849 & 2528.5375 & \multirow{2}{*}{98.99} \\
\hline & Unsegmented & \begin{tabular}{|l|}
170.8582 \\
\end{tabular} & 1645 & 738.3848 & 2554.2435 & \\
\hline \multirow{2}{*}{$\begin{array}{c}\text { Image } \\
2\end{array}$} & Segmented & 165.6944 & 1562 & 900.4324 & 2628.1268 & \multirow{2}{*}{99.43} \\
\hline & Unsegmented & 160.2324 & 1533 & 949.756 & 2642.9884 & \\
\hline \multirow{2}{*}{$\begin{array}{c}\text { Image } \\
3 \\
\end{array}$} & Segmented & 187.535 & 1220 & 903.5578 & 2311.0928 & \multirow{2}{*}{98.35} \\
\hline & Unsegmented & 175.876 & 1252 & 921.7996 & 2349.6756 & \\
\hline \multirow{2}{*}{$\begin{array}{c}\text { Image } \\
4\end{array}$} & Segmented & \begin{tabular}{|l|}
185.809 \\
\end{tabular} & 1332 & 726.4809 & 2244.2899 & \multirow{2}{*}{98.82} \\
\hline & Unsegmented & 191.179 & \begin{tabular}{|l|}
1199 \\
\end{tabular} & 880.71 & 2270.8890 & \\
\hline \multirow{2}{*}{$\begin{array}{c}\text { Image } \\
5\end{array}$} & Segmented & 192.5944 & 1458 & 878.1424 & 2528.7368 & \multirow{2}{*}{98.43} \\
\hline & Unsegmented & \begin{tabular}{|l|}
135.5644 \\
\end{tabular} & 1509 & 924.442 & 2569.0064 & \\
\hline & & & & & Average: & \\
\hline
\end{tabular}

Table 2. Segmentation accuracy based on number of objects

Table 3. Segmentation results for different images using proposed methodology

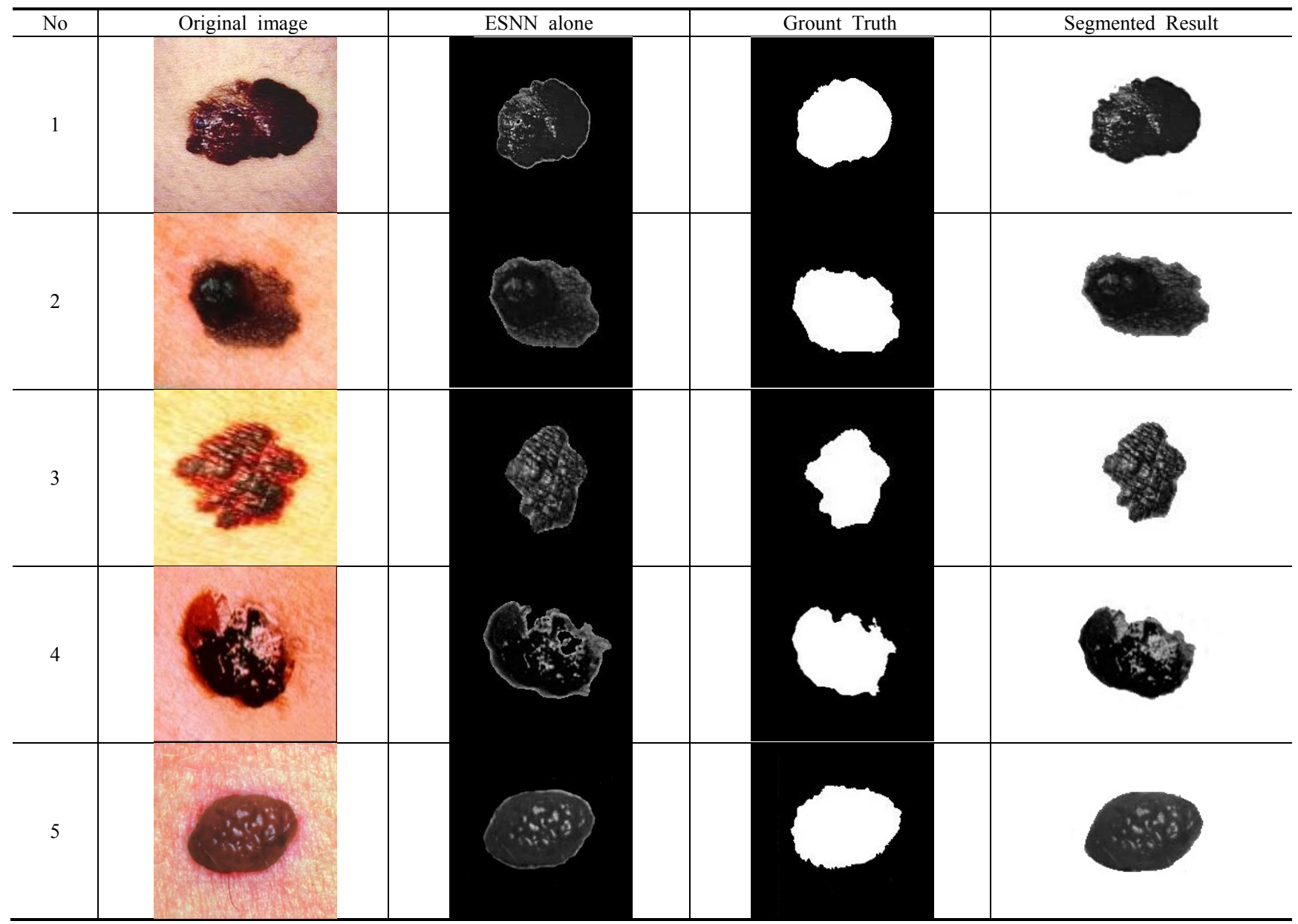


Table 4. Performance comparison of various segmentation techniques

\begin{tabular}{c|c|c|c}
\hline Existing Methods & Sensitivity & Specificity & Accuracy \\
\hline SRM [8] & $89.4 \%$ & $92.7 \%$ & $92.3 \%$ \\
\hline Otsu-R [9] & $87.3 \%$ & $85.4 \%$ & $84.9 \%$ \\
\hline Otsu-RGB [10] & $93.6 \%$ & $80.3 \%$ & $80.2 \%$ \\
\hline Otsu-PCA [6] & $79.6 \%$ & $99.6 \%$ & $98.1 \%$ \\
\hline TDLS [1] & $91.2 \%$ & $99.0 \%$ & $98.3 \%$ \\
\hline Proposed Method & $90.6 \%$ & $99.2 \%$ & $98.8 \%$ \\
\hline
\end{tabular}

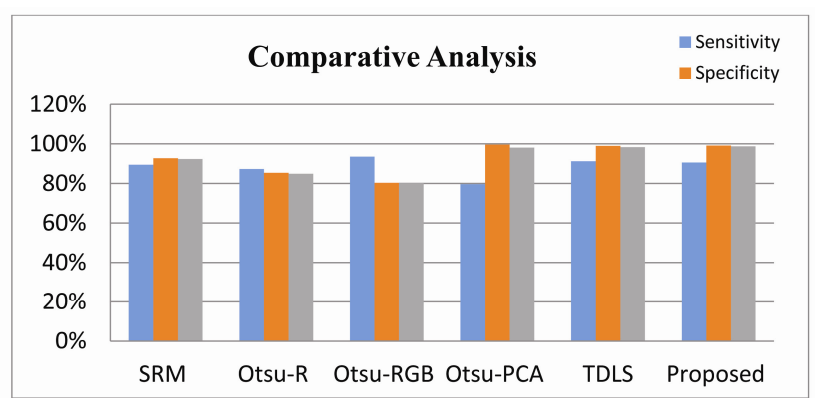

Fig. 3. Comparative analysis of various segmentation techniques

\subsection{Segmentation performance in terms of number of objects}

The segmentation accuracy based on the number of objects $[11,29]$ is explained in this section. Segmentation accuracy based on number of objects $A_{\text {prop }}$ is computed as follows

$$
\begin{aligned}
& A_{\text {prop }}=\frac{S_{\text {prop }}}{O_{\text {prop }}} \times 100 \\
& S_{\text {prop }}=\left(\text { Solidity }+ \text { Area }+ \text { Perimeter }_{\text {segmented }}\right) \\
& O_{\text {prop }}=\left(\text { Solidity }+ \text { Area }+ \text { Perimeter }_{\text {unsegmented }}\right)
\end{aligned}
$$

where $S_{\text {prop }}$ and $O_{\text {prop }}$ are the region properties of the segmented and unsegmented images respectively.

Table 1 shows the segmentation accuracy based on Sensitivity, Specificity and Accuracy and Table 2 shows the segmentation accuracy based on number of objects for all skin lesion images. From these tables, it can be observed that our proposed segmentation method provides an overall accuracy of $98.8 \%$ in segmenting the skin lesion images effectively.

\section{Comparative Analysis}

The proposed algorithm is compared with different lesion segmentation algorithms. Table 4 shows the detailed comparison of segmentation accuracy of the proposed method with five existing lesion segmentation techniques. The first algorithm (L-SRM) [8]. The second algorithm is (Otsu-RGB) [9], the third algorithm is (Otsu-PCA) the fourth algorithm is (TDLS) [1]. The segmentation algorithms are compared to manually segmented ground truth. The proposed algorithm compared visually and by calculating sensitivity, specificity, accuracy and also based on the number of pixels of the image to find the accuracy. From the table, it can be observed that the segmentation accuracy of the proposed methodology is higher when compared to the other existing methods for segmenting skin lesions

\section{Conclusions and Future Work}

In this paper, a new texture based neural network approach has been proposed for segmenting the skin lesion images effectively. The major advantage of using the Echo state Neural Network is to overcome the limitations of existing skin lesion segmentation techniques since it combines the textural features of different pixels present in the entire image and also increases the accuracy of segmentation. The combination of texture along with Neural Networks is used for entire area of the image. In order to measure the performance of the proposed method, the proposed algorithm is trained and tested for 862 different skin slices. Overall accuracy of the proposed method is compared with five different skin lesion image segmentation techniques. The major contribution of this paper is an improvement in segmentation accuracy by combining neural network along with the texture features. Future works in this direction can be the proposal of a new methodology for classifying the different types of lesions and to analyze the time complexity of it.

\section{References}

[1] Jeffrey Glaister, Alexander Wong, David A. Clausi, "Segmentation of Skin Lesions From Digital Images Using Joint Statistical Texture Distinctiveness", IEEE Transactions on Biomedical Engineering, Vol. 61, NO. 4, April 2014.

[2] Jemal, M. Saraiya, P. Patel, S. S. Cherala, J. Barnholtz-Sloan, J. Kim, C. L. Wiggins, and P. A. Wingo, "Recent trends in cutaneous melanoma incidence and death rates in the united states, 19922006," J. Amer. Acad. Dermatol., vol. 65, no. 5, pp. S17.e1-S17.e11, Nov. 2011.

[3] Celebi ME, Aslandogan YA, Stoecker WV, Iyatomi H, Oka H, Chen X. Unsupervised border detection in dermoscopy images. Skin Res Technol 2007; 13:1-9.

[4] R. Harralick, K. Shanmugam, Dinstein, "Textural Features for Image Classification", IEE Trsans on System, Man and Cybernetics, Vol. 3, No. 6, 1973, pp. 610-621.

[5] S. Hwang and M. E. Celebi, "Texture segmentation of dermoscopy images using Gabor filters and gmeans clustering," in Proc. Int. Conf. Image Process., Comput. Vision, Pattern Recog, Jul. 2010, 
pp. $882-886$.

[6] DermQuest, (2012). [Online]. Available-: http://www.mquest.com

[7] Cavalcanti PG, Scharcanski J, Baranoski GV. A twostage approach for discriminating melanocytic skin lesions using standard cameras. Expert Syst Appl 2013; 40: 4054-64.

[8] M. E. Celebi, H. A. Kingravi, H. Iyatomi, Y. A. Aslandogan, W. V. Stoecker, R. H. Moss, J. M. Malters, J. M. Grichnik, A. A. Marghoob, H. S. Rabinovitz, and S. W. Menzies, "Border detection in dermoscopy images using statistical region merging," Skin Res. Technol., vol. 14, no. 3, pp. 347-353, 2008.

[9] P. G. Cavalcanti, J. Scharcanski, and C. B. O. Lopes, "Shading attenuation in human skin color images," in Advances in Visual Computing, (ser. Lecture Notes in Computer Science), vol. 6453 Heidelberg, Germany: Springer, 2010, pp. 190-198.

[10] Glowacz A, Glowacz A, Glowacz Z. Recognition of monochrome thermal images of synchronous motor with the application of skeletonization and classifier based on words. Archives of Matallurgy and Materials 2015.

[11] P. G. Cavalcanti and J. Scharcanski, "Automated prescreening of pigmented skin lesions using standard cameras," Comput.Med. Imag. Graphics, vol. 35, no. 6, pp. 481-491, Sep. 2011.

[12] Faizal Khan, Z \& Kannan, "Intelligent Approach for Segmenting CT Lung Images Using Fuzzy Logic with Bitplane", Journal of Electrical Engineering and Technology, Vol. 9, No. 4, pp-742-752, 2014

[13] Claudio Gallicchio. Alessio Micheli, "Tree Echo State Networks", Neuro computing, Vol. 101, no. 4, pp. 319-337, 2013.

[14] Petrènas A' Marozas V, Sörnmo L, Lukosevicius A, "An echo state neural network for QRST cancellation during atrial fibrillation", IEEE Trans Biomed Eng, vol. 59, no. 10, pp. 2950-2957, 2012.

[15] Jisha Mariyam John, Simi Susan Samuel, Neethu Maria John, Segmentation of Skin Lesions from Digital Images using Texture Distinctiveness with Neural Network, International Journal of Advanced Research in Computer and Communication Engineering Vol. 3, Issue 8, August 2014.

[16] Degan Zhang, Xuejing Kang. "A novel image denoising method based on spherical coordinates system, EURASIP Journal on Advances in Signal Processing, pp. 1-10, 2012.

[17] Zhu W, Zeng N, Wang N. Sensitivity, specificity, accuracy, associated confidence interval and ROC analysis with practical $\mathrm{SAS} \AA$ implementations. In: Proceedings of NESUG: Health Care and Life Sciences; 2010 Nov, 14-17. Baltimore, Maryland; 2010 .
[18] W. V. Stoecker, C.-S. Chiang, and R. H. Moss, "Texture in skin images: Comparison of three methods to determine smoothness," Comput. Med. Imag Graphics, vol. 16, no. 3, pp. 179-190, 1992.

[19] L. Xu, M. Jackowskia, A. Goshtasby, D. Roseman, S. Bines, C. Yu, A. Dhawan, and A. Huntley, "Segmentation of skin cancer images," Image Vis. Comput., vol. 17, pp. 65-74, 1999.

[20] S. Hwang and M. E. Celebi, "Texture segmentation of dermoscopy images using Gabor filters and gmeans clustering," in Proc. Int. Conf. Image Process., Comput. Vision, Pattern Recog, Jul. 2010, pp. 882886.

[21] Argenziano G, Soyer HP, De Giorgi V, Piccolo D, Carli P, Delfino M, et al. Dermoscopy: ATutorial. Milan: EDRA Medical Publishing \& NewMedia; 2002.

[22] Menzies SW, Bischof L, Talbot H, Gutenev A, Avramidis M, Wong L. The performance of Solar Scan: An automated dermoscopy image analysis instrument for the diagnosis of primary melanoma. Arch Dermatol 2005;141(11):1388-96.

[23] Stolz W, Riemann A, Cognetta AB, et al., ABCD rule of dermatoscopy: a new practical method for early recognition of malignant melanoma, Eur J Dermatol, 1994; 4:521-7.

[24] Nachbar F, Stolz W, Merkle T, et al., The ABCD rule of dermatoscopy. High prospective value in the diagnosis of doubtful melanocytic skin lesions, J Am Acad Dermatol, 1994; 30: 551-559.

[25] Glowacz A. Recognition of Acoustic Signals of Synchronous Motors with the Use of MoFS and Selected Classifiers. Measurement Science Review 2015; 15 (4): 167-175.

[26] Z. Faizal khan, S. Veeramalai, G.Nalini priya, M. Ramesh kumar, A. Naresh kumar. A. Kannan, “A novel Approach for Segmenting Computer Tomography Lung Images Using Echo State Neural Networks", Journal of Theoretical and Applied Information technology, Vol. 68, No. 3, October 2014.

[27] Faizal Khan, Z, Automated Segmentation of Skin Lesions using Seed Points and scale Invariant Semantic Mathematic Model, Advances in Intelligent systems and Computing, Sprinjer, pp. 219-227.

[28] Degan Zhang, Xiang Wang, Xiaodong Song. "New Medical Image Fusion Approach with Coding Based on SCD in Wireless Sensor Network", Journal of Electrical Engineering \& Technology, 2015, 10 (6): 2384-2392.

[29] Faizal Khan, Z \& Kannan, "Intelligent Segmentation of Medical images using Fuzzy Bitplane Thresholding", "Measurement science and Review, Vol. 14, No. 2, pp. 94-101, 2014. 


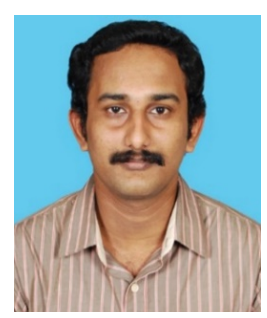

Z. Faizal Khan, received obtained a Doctor of Philosophy (Ph.D.) in Information and Communication Engineering from Anna University Chennai, Tamil Nadu, India. He received a Bachelor of Engineering from Anna University Tirunelveli and Master's Degree in Computer Science and Engineering from Anna University Chennai. He is presently working as an Assistant professor in the Department of Computer and Network Engineering, College of Engineering, Shaqra University, Kingdom of Saudi Arabia. His experience and areas of interest focus on Medical image processing, Pattern recognition, Soft computing and Bio-informatics.

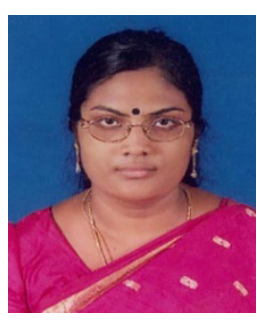

Nalinipriya Ganapathi obtained a Doctor of Philosophy (Ph.D.) in Information and Communication Engineering from Anna University Chennai, Tamil Nadu, India. She received a Bachelor of Engineering from Madras University and Master's Degree in Computer Science and Engineering from Anna University Chennai. She is presently working as a professor in the Department of Information Technology, Saveetha Engineering College, Chennai, Tamilnadu., India. Her experience and areas of interest focus on Medical Data Mining, Cloud security, Software engineering, Soft computing Wireless networks, and Bio-informatics. 